\title{
Bigger Buffer $k$-d Trees on Multi-Many-Core Systems
}

\author{
Fabian Gieseke $^{1}$, Cosmin Eugen Oancea ${ }^{2}$, Ashish Mahabal ${ }^{3}$, \\ Christian Igel ${ }^{2}$, and Tom Heskes ${ }^{1}$ \\ 1 - Institute for Computing and Information Sciences - Radboud University Nijmegen \\ Toernooiveld 212, 6525 EC Nijmegen - The Netherlands \\ $\{$ f.gieseke, t.heskes\}@cs.ru.nl \\ 2 - Department of Computer Science - University of Copenhagen \\ Universitetsparken 5, 2100 Copenhagen - Denmark \\ $\{$ cosmin.oancea, igel $\} @$ di.ku.dk \\ 3 - Caltech Astronomy - Caltech \\ 1200 East California Blvd, Pasadena CA 91125 - USA \\ aam@astro.caltech.edu
}

\begin{abstract}
A buffer $k$-d tree is a $k$-d tree variant for massively-parallel nearest neighbor search. While providing valuable speed-ups on modern many-core devices in case both a large number of reference and query points are given, buffer $k$-d trees are limited by the amount of points that can fit on a single device. In this work, we show how to modify the original data structure and the associated workflow to make the overall approach capable of dealing with massive data sets. We further provide a simple yet efficient way of using multiple devices given in a single workstation. The applicability of the modified framework is demonstrated in the context of astronomy, a field that is faced with huge amounts of data.
\end{abstract}

\section{Motivation}

Nearest neighbor search is a fundamental problem and an ingredient of many state-of-the-art data analysis techniques. While being a conceptually very simple task, the induced computations can quickly become a major bottleneck in the overall workflow when both a large reference and a large query set are given. In the literature, many techniques have been proposed that aim at accelerating the search. Typical are include the use of spatial search structures, approximation schemes, and parallel implementations [12/5/1014/18. A recent trend in the field of big data analytics is the application of massively-parallel devices such as graphics processing units (GPUs) to speed up the involved computations. While such modern many-core devices can significantly reduce the practical runtime, obtaining speed-ups over standard CPU-based execution is often not straightforward and usually requires a careful adaptation of the sequential implementations.

Spatial search structures such as $k$-d trees are an established way to reduce the computational requirements induced by nearest neighbor search for spaces of moderate dimensionality (e.g., up to $d=30$ ). A typical parallel $k$-d tree 
based search assigns one thread to each query and all threads process the same tree simultaneously. Such an approach, however, is not suited for GPUs since each thread might induce a completely different tree traversal, which results in massive branch divergence and irregular accesses to the device's memory.

Recently, we have proposed a modification of the classical $k$-d tree data structure, called buffer $k$ - $d$ tree, which aims at combining the benefits of both spatial search structures and massively-parallel devices [11]. The key idea is to assign an additional buffer to each leaf of the tree and to delay the processing of the queries reaching a leaf until enough work has been gathered. In that case, all queries stored in all buffers are processed together in a brute-force manner via the many-core device. While the framework achieves significant speed-ups on modern many-core devices over both a massively-parallel brute-force execution on GPUs as well as over a multi-threaded $k$-d tree based search running on multi-core systems, it is limited by the amount of reference and query points that fit on a GPU. In this work, we show how to remove this limitation by modifying the induced workflow to efficiently support huge reference and query point sets that are too large to be completely stored on the devices. This crucial modification renders buffer $k$-d trees capable of dealing with huge data sets.

\section{Background}

For the sake of completeness, we provide the background related to massivelyparallel programming on GPUs as well as to classical $k$-d tree-based nearest neighbor search. We further sketch the key ideas of the buffer $k$-d tree extension.

\subsection{Architecture and Programming Model}

Modern many-core devices such as GPUs offer massive parallelism and can nowadays also be used for so-called general-purpose computations such as matrixmatrix multiplication. In contrast to standard CPU-based systems, GPUs rely on simplified control units and on a memory subsystem that does not attempt to provide the illusion of a uniform access cost to memory.

GPU architectures are typically formed from a number of vector processors, several special function units and an amount of fast memory that is split between registers, L1 and L2 data caches, scratchpad and read-only memory. Each vector processor consists of multiple execution units that execute in lock step, i.e., in a single-instruction multiple data (SIMD) fashion, and each execution unit is further multi-threaded in order to hide memory latency.

The GPU programming model typically reflects this hardware organization: For example, expressing a parallel computation in OpenCL 22] requires the user to write a kernel that will be run simultaneously by many threads. Threads are grouped into workgroups, which are run on the same vector processor, called streaming multiprocessor (SM). Programmers need to explicitly declare in what memory the data is stored. Further, they can use fast synchronization and com-

munication within a workgroup via local memory. For NVIDIA GPUs, groups of 32 threads execute in a SIMD fashion; such a group is called warp. 
The main ingredients for an efficient many-core implementation are (1) exposing sufficient parallelism to fully utilize the device and (2) accessing the memory in an efficient way. The latter one includes techniques that

(a) hide the latency of the memory transfer between host and GPU, for example by overlapping the kernel execution with the memory transfer, and

(b) restructure the program in order to improve both spatial and temporal locality of reference to the global memory of the GPU 1

\subsection{Massively-Parallel Nearest Neighbor Computations}

We address the problem of computing the $k \geq 1$ nearest neighbors of all points given in a query set $Q=\left\{\mathbf{q}_{1}, \ldots, \mathbf{q}_{m}\right\} \subset \mathbb{R}^{d}$ w.r.t. to all points provided in a reference set $P=\left\{\mathbf{x}_{1}, \ldots, \mathbf{x}_{n}\right\} \subset \mathbb{R}^{d}$. Usually, the "closeness" between two points is defined via the Euclidean distance (which we will use), but other distance measures can be applied as well. Such nearest neighbor computations form the basis for a variety of methods both in data mining and machine learning including proximity-based outlier detection, classification, regression, density estimation, and dimensionality reduction, see, e.g., Hastie et al. [12]. The task of computing the induced distances (and keeping track of the list of neighbors per object) can be addressed naively in a brute-force manner spending $\varnothing(n m \cdot(d+\log k))$ time, which quickly becomes computationally very demanding. Massively-parallel computing can significantly reduce the runtime in this case as shown by Garcia et al. 10. Still, for large query and reference sets, the computational requirements can become very large.

Various other approaches have been proposed in the literature that aim at taking advantage of the computational resources provided by GPUs in combination with other techniques 4 1823. The focus of this work is on massivelyparallel processing of $k$-d trees. While several implementations have been proposed that address such traversals from a more general perspective (e.g., in the context of ray tracing) [13,17]20]26, these approaches are not suited for nearest neighbor search in moderate-sized feature spaces (i.e., $d>3$ ), except for the recently proposed buffer $k$-d tree extension [11].

\subsection{Nearest Neighbor Search via $K$-d Trees}

Spatial search structures such as $k$-d trees can be used to speed up nearest neighbor search. $K$-d trees can be constructed as follows [19]: For a given point

\footnotetext{
${ }^{1}$ Here, spatial locality corresponds to the case in which threads in the same warp access consecutive global memory locations in the same instruction. Such a coalesced access is collapsed into a single global memory transaction (in contrast, if threads in a warp access memory with a stride of 32 , then 32 sequential memory transaction are needed). Temporal locality corresponds to the case in which most of the threads in the same workgroup access the same memory location in the same instruction. In this case, the first warp accessing it brings the corresponding data block to the L1 cache within one memory transfer (which is then used by the subsequent warps).
} 
set $P$, a $k$-d tree is a binary tree with the root corresponding to $P$. The children of the root are obtained by splitting the point set into two (almost) equal-sized subsets, which are processed recursively. In their original form, $k$-d trees are obtained by resorting to the median values in dimension $i \bmod d$ to split a point set corresponding to a node $v$ at level $i$ (starting with the root at level 0 ) 2 The recursive process stops as soon as a predefined number of points are left in a subset. The $k$-d tree stores the splitting values in its internal nodes; the points corresponding to the remaining sets are stored in the leaves.

The tree structure can be used to accelerate nearest neighbor search: Let $\mathbf{q} \in \mathbb{R}^{d}$ be a query point. For the sake of exposition, we focus on $k=1$ (the case of $k>1$ neighbors works similarly). The overall search takes place in two phases. In the first one, the tree is traversed from top to bottom to find the $d$ dimensional cell (induced by the tree/splitting process) that contains the query point q. Going down the tree can be conducted efficiently using the median values stored in the internal nodes of the $k$-d tree. In the second phase, the tree is traversed bottom-up, and on the way back to the root, subtrees are checked in case the query point is close to the corresponding splitting hyperplane. If the distance of the query point $\mathbf{q}$ to the hyperplane is less than the distance to the current nearest neighbor candidate, then the subtree is checked for better candidates (recursively). Otherwise, the whole subtree can be safely pruned (no recursion). Once the root is reached twice, the overall process stops and the final nearest neighbor candidate is returned.

Given a low-dimensional search space, it is usually sufficient to process a relatively small number of leaves, which results in a logarithmic runtime behavior (i.e., $\varnothing(\log n)$ time per query in practice). However, the performance usually decreases for increasing $d$ due to the curse of dimensionality. In the worst case, all nodes and leaves of the $k$-d tree need to be processed, which yields a linear query time (i.e., $\varnothing(n)$ time per query).

\subsection{Revisited: Buffer $k$-d Trees}

A standard multi-threaded $k$-d tree-based traversal assigns one thread to each query. For GPUs, such an approach is not suited since each thread might induce a completely different path, which significantly shortens their computational benefits. The main idea of the buffer $k$-d tree extension is to delay the processing of the queries by buffering similar patterns prior to their common processing [11. The reorganized workflow is based on buffer $k$-d trees, which are sketched next, followed by a description of the buffered nearest neighbor search.

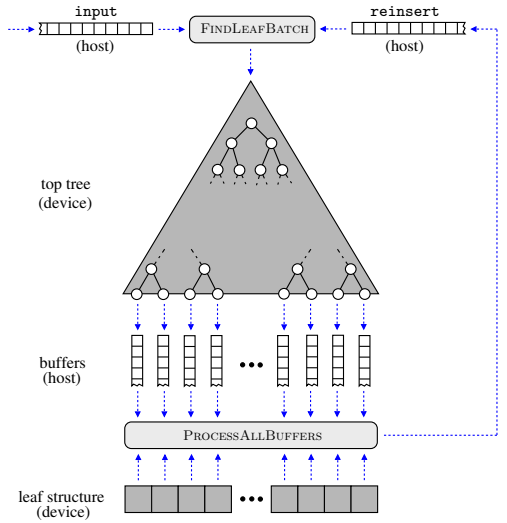

Figure 1: A buffer $k$-d tree [11: The gray elements are stored on GPU.

\footnotetext{
${ }^{2}$ Other splitting rules might be applied (e.g., according to the "longest" side).
} 


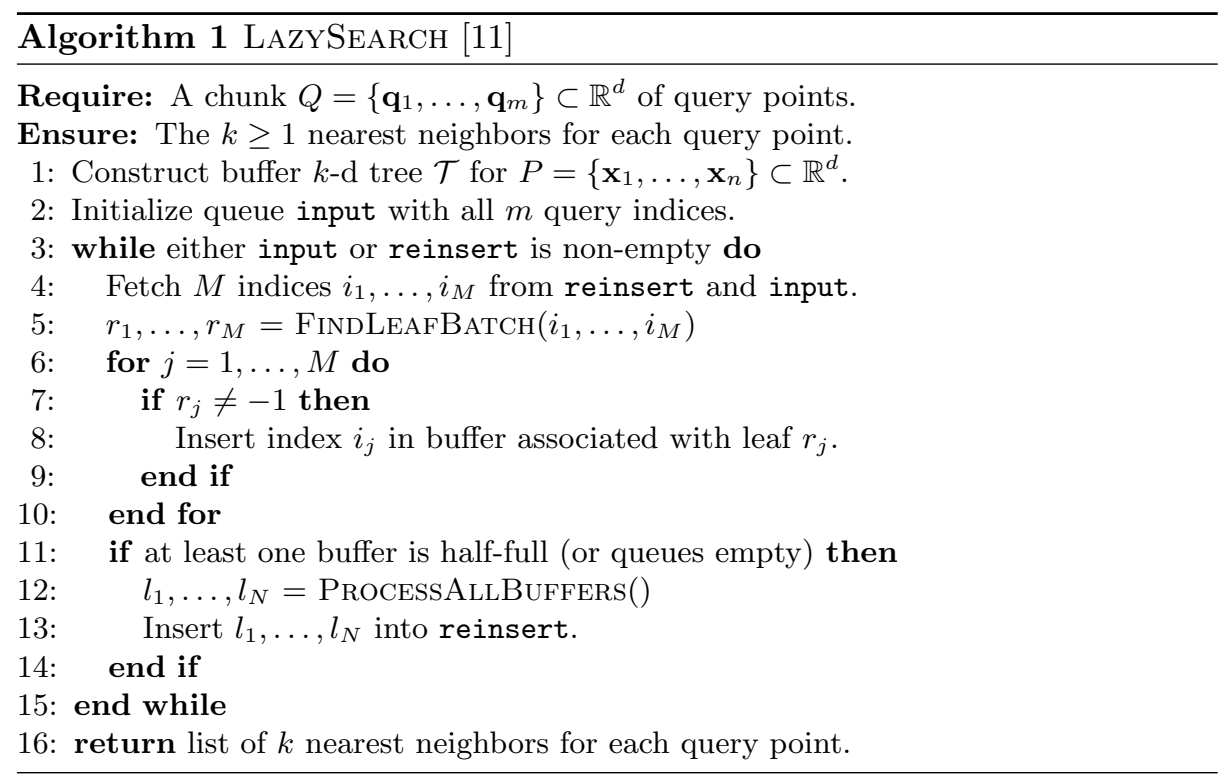

A buffer $k$-d tree consists of (1) a top tree, (2) a leaf structure, (3) a set of buffers, and (4) two input queues that store the queries, see Figure 1 . The top tree corresponds to a classical $k$-d tree with its splitting values (e.g., medians) laid out in memory in a pointer-less manner. The leaf structure stores the point sets that stem from the splitting process in a consecutive manner. In addition, a buffer is attached to each leaf of the top tree that can store $B$ query indices (e.g., $B=1024$ ). The input queues are used to store the input query indices and the query indices that need further processing after a ProcessAllBuffers call.

A buffer $k$-d tree can be used to delay the processing of the queries by performing several iterations, see Algorithm 11: In each iteration, the procedure FINDLEAFBATCH retrieves indices from both the input and reinsert queue and propagates them through the top tree. The indices, which are stored in the corresponding buffers, are processed in chunks via the procedure ProcessAllBuffers once the buffers get full. All indices that need further processing (i.e., their implicit tree traversal has not reached the root twice) are inserted into reinsert again. Thus, in each iteration, one (1) finds the leaves that need to be processed next and (2) updates the queries' nearest neighbors.

While the first phase is not well-suited for massively-parallel processing, the second one is and, since it constitutes the most significant part of the runtime, yields valuable overall speed-ups. The main advantage of the reorganized workflow is that all queries are processed in the same block-wide SIMD instruction and exhibit either good spatial or temporal locality of reference, i.e., coalesced or cached global memory accesses (see Section 2.1). For details of the particular many-core implementation of the procedure PROCESSAllBuffers, we refer to Gieseke et al. [11. Note that both the top tree and the leaf structure need to 
be stored on the GPU given the original implementation [11] - this limits the amount of reference patterns that can be processed.

\section{$3 \quad$ Processing Bigger Trees}

One issue not addressed so far is the fact that the memory of modern GPUs is still relatively small compared to host memory ${ }^{3}$ This limits the amount of data points that can be processed. We now describe modifications that allow the buffer $k$-d trees to scale to massive data sets not fitting on a GPU anymore.

\subsection{Construction Phase}

As shown below, one can basically process arbitrarily large query sets by considering chunks of data points. Dealing with huge reference sets, however, is more difficult: Since the top tree and the full leaf structure (that stores the rearranged reference points) have to be made available to all threads during the execution of Process AllBuffers, one cannot directly split up the leaf structure. However, as explained next, one can avoid storing the leaf structure in its full entirety on the many-core device without significantly increasing the overall runtime.

We start by focusing on the space needed for the top tree: From a practical perspective, a small top tree is usually advantageous compared to the full tree used by a classical $k$-d treebased search, since the efficiency gain on GPUs stems from processing "big" leaves. For instance, given a reference set $P \subset \mathbb{R}^{10}$ with two million points, top trees of height $h=8$ or $h=9$ are usually optimal [11. Further, since only median values are stored in the top tree, the space consumption is negligible even given much bigger top trees 4 In addition, the top tree can be built efficiently via linear-time median finding [3], which results in $\varnothing(h \log n)$ time for the whole construction phase. Hence, one can (1) build the top tree

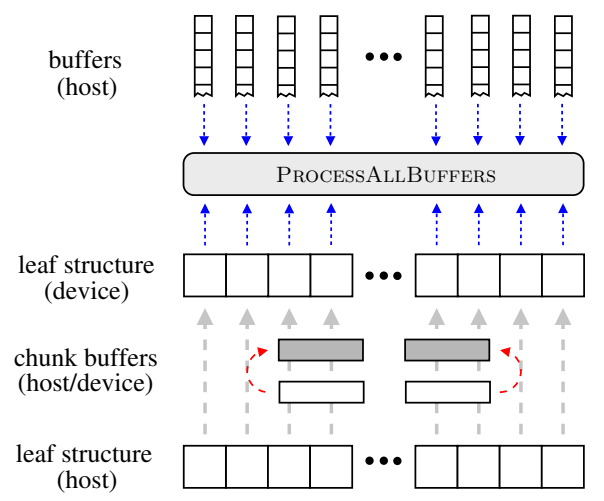

Figure 2: Adapted memory layout: The leaf structure is not stored explicitly on the device. Instead, memory for two chunk buffers is allocated on both the device and the host, which is used for an concurrent computeand-copy processing of the leaf structure. efficiently on the host system and (2) store it in its full entirety on the GPU.

The main space bottleneck stems from the leaf structure. For instance, one billion points in $\mathbb{R}^{15}$ occupy about 60 gigabytes of space- too much for a modern

\footnotetext{
${ }^{3}$ We assume that the host's memory is large enough to store the whole buffer $k$-d tree.

${ }^{4}$ For a tree of height $h=20$, suitable for more than one billion points, less than ten megabytes are needed. Note that the space for the buffers (e.g., of size 128 each) stored on the host does usually not cause any problems (e.g., less then a gigabyte).
} 
many-core device. For this reason, we do not copy the leaf structure from the host to the device after the construction of the top tree. Instead, we allocate space for two chunk buffers of fixed size on the many-core device. These chunks will be used to overlap the execution of the Process AllBuffers procedure with the host-to-device memory transfer for the next chunk. Note that we also allocate two associated memory buffers on the host (pinned memory 22]) to achieve efficient concurrent compute and copy operations. The overall memory layout is shown in Figure 2, where the memory that is actually allocated on the device is sketched via gray rectangles.

\subsection{Query Phase}

We now describe the details of the modified querying process. The idea is to keep the leaf structure on the host system and to process the buffers via chunks with concurrent compute and copy operations.

Processing the Leaf Structure: In each iteration of Algorithm 1, the procedure Process AllBuffers is invoked to retrieve all query indices from the buffers attached to the leaves. The queries are processed in a massively-parallel fashion, where each thread compares a particular query with all reference points stored in the associated leaf. Given the modified memory layout, one can now process the leaves in chunks in the following way: The leaf structure containing all $n$ rearranged reference points is split into $1<N \ll n$ chunks $C_{1}, \ldots, C_{N}$ (e.g., $N=10$ ). Each chunk $C_{j}$ contains the points of the leaf structure at positions $k=C_{j}^{L}, \ldots, C_{j}^{R}$, where $C_{j}^{L}=\left\lceil\frac{(j-1) \cdot n}{N}\right\rceil$ and $C_{j}^{R}=\left\lceil\frac{j \cdot n}{N}\right\rceil$. A buffer attached to the top tree corresponds to a leaf in the leaf structure with leaf bounds $0 \leq l_{i}<r_{i} \leq n-1$. All queries removed from the buffers are then processed in $N$ iterations and a query $i$ with leaf bounds $l_{i}$ and $r_{i}$ is processed in iteration $j \in\{1, \ldots, N\}$ if $\left[l_{i}, r_{i}\right] \cap\left[C_{j}^{L}, C_{j}^{R}\right] \neq \emptyset$, i.e., if the leaf bounds overlap with chunk $C_{j}$.

The chunks are processed sequentially $C_{1}, C_{2}, \ldots, C_{N}$. The data needed for each chunk is copied from the host to one of the two chunk buffers allocated on the device prior to conducting the brute-force computations, see Figure 2 , To hide the overhead induced for these copy operations, the copy process for the next chunk is started as soon as the computations for its predecessor have been invoked. In particular, the processing of a chunk $C_{j}$ takes place in three phases:

(1) Brute: First, the massively-parallel brute-force nearest neighbor computations are invoked (non-blocking kernel call). The data needed for these computations (chunk $C_{j}$ ) have been copied in round $j-1$ (for $C_{0}$, the data is either available from an initial copy operation or from the previous round).

(2) Copy: While the brute-force computations are conducted by the GPU, the data for the next chunk are copied from host to the buffer on the device that is currently not in use $5^{5}$ Note that copy operations on the host system have

\footnotetext{
${ }^{5}$ For $j=N$, the data for chunk 0 are copied from host to the corresponding buffer on the device for next round (i.e., next call of ProcessAllBuffers).
} 
to be conducted as well to ensure that the correct part of the leaf structure is moved to the appropriate pinned memory buffer (which is then copied to the device), see again Figure 2

(3) Wait: In the final phase, one simply waits for the kernel invoked in the first phase to finish its computations (blocking call).

The iterative compute-and-copy processing of the chunks can be implemented via two (OpenCL) command queues [22]: For the first chunk, phase (1) and (3) are instantiated via command queue A, whereas the copy process (2) is instantiated via command queue B (non-blocking for both (1) and (2)). For the second chunk, phases (1) and (3) are instantiated via command queue B and (2) via command queue A. This process continues until all chunks have been processed. In essence, the use of two command queues allows the copy phase (2) to run in parallel with the brute-force computation phases (1) and (3).

Given the new workflow, one can basically handle an arbitrary amount of reference patterns. The only restriction is the memory available on the host. In case not enough main memory is available, one can store the leaf structure on disk and copy the chunks from disk to device memory (via host memory) 6

Multi-Many-Core Querying: Assuming that a fixed amount of memory is available on the many-core device to store the query patterns and the results at all times (e.g., one gigabyte), one can process an arbitrarily large query set by removing fully processed indices and by adding new indices on-the-fly, see Algorithm 1. An even simpler approach is to split up the queries into chunks and to handle these chunks independently. One drawback of the latter approach could be the overhead induced by applying the procedure ProcessAllBuffers in case the buffers are not sufficiently filled (which usually takes place as soon as no queries are available anymore). However, given relatively large chunks, the induced overhead is very small as shown in our experimental evaluation.

In a similar fashion, one can make use of multiple many-core devices by splitting all queries into "big" chunks according to the devices that are available. These chunks, which might have to be split into smaller chunks as described above, can be processed independently from each other.

\section{Experiments}

The purpose of the experiments provided below is to analyze the efficiency of the modified workflow and to sketch the potential of the overall approach in the context of large-scale scenarios. For a detailed experimental comparison including an analysis of the different processing phases and the influence of parameters related to the buffer $k$-d tree framework, we refer to our previous work [11.

\footnotetext{
${ }^{6}$ Depending on the particular architecture, the induced copying processes might become a major bottleneck. However, one can shorten this drawback by increasing the leaf size of the buffer $k$-d tree such that more computations have to be conducted for each transfer of data from disk to device.
} 


\subsection{Experimental Setup}

All runtime experiments were conducted on a standard desktop computer with an Intel(R) Core(TM) i7-4790K CPU running at 4.00GHz (4 cores; 8 hardware threads), 32GB RAM, and two Nvidia GeForce Titan Z GPUs (each consisting of two devices with 2880 shader units and 6 GB main memory). The operating system was Ubuntu 14.4.3 LTS (64 Bit) with kernel 3.13.0-52, CUDA 7.0.65 (graphics driver 340.76), and OpenCL 1.2. All algorithms were implemented in C and OpenCL, where Swig was used to obtain appropriate Python interfaces $]^{7}$ The code was compiled using gcc-4.8.4 at optimization level -03.

For the experimental evaluation, we report runtimes for both the construction and the query phase (referred to as "train" and "test" phases), where the focus is on the latter one (that makes use of the GPUs). We consider the following three implementations:

(1) bufferkdtree(i): The adapted buffer $k$-d tree implementation with both FindLeafBatch and ProcessAllBuffers being conducted on $i$ GPUs.

(2) kdtree(i): A multi-core implementation of a $k$-d tree-based search, which runs $i$ threads in parallel on the CPU (each handling a single query).

(3) brute (i): A brute-force implementation that makes use of $i$ GPUs to process the queries in a massively-parallel manner.

The parameters for the buffer $k$-d tree implementation were fixed to appropriate values ${ }^{8}$ Note that both competitors of bufferkdtree have been evaluated extensively in the literature; the reported runtimes and speed-ups can thus be put in a broad context. For simplicity, we fix the number $k$ of nearest neighbors to $k=10$ for all experiments.

We focus on several data-intensive tasks from the field of astronomy. Note that a similar runtime behavior can be observed on data sets from other domains as well as long as the dimensionality of the search space is moderate (e.g., from $d=5$ to $d=30$ ). We follow our previous work and consider the psf mag, psd_model_mag, and all_mag data sets of dimensionality $d=5, d=10$, and $d=15$, respectively; for a description, we refer to Gieseke et al. [11. In addition, we consider a new dataset derived from the Catalina Realtime Transient Survey (crts) [76[16]. This survey contains tens to hundreds of observations for more than 500 million sources over a large part of the sky. The resulting light-curves (time-series of light received as a function of time) are used to derive several statistical features ${ }^{9}$ We use ten such features on a set of 30 million light-curves for the experiments described here. The main interest is to find outliers in the large space that can lead to interesting discoveries.

\footnotetext{
7 The code is publicly available under https://github.com/gieseke/bufferkdtree.

${ }^{8}$ For a tree of height $h$, we fixed $B=2^{2 \dot{4}-\dot{n}}$ and the number $M$ of indices fetched from input and reinsert in each iteration of Algorithm 1 to $M=10 \cdot B$. Note that the particular assignments for these and other parameters did not have a significant influence on the performance as long as they were set to reasonable values.

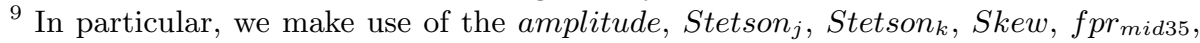
$f p r_{\text {mid50 }}, f p r_{\text {mid65 }}, f p r_{\text {mid80 }}$, shov, maxdiff [218].
} 


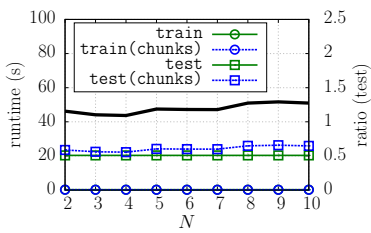

(a) $n=500,000$

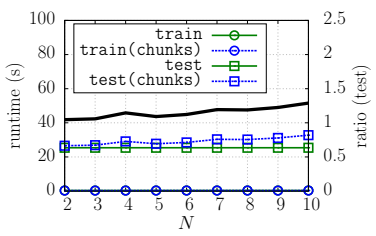

(b) $n=1,000,000$

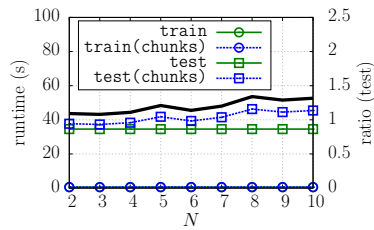

(c) $n=2,000,000$

Figure 3: Comparison of the training and testing times between the original workflow (train and test) and the modified workflow that processes the leaves in chunks (train(chunks) and test(chunks)). A varying number $N$ of chunks and training points $n$ are considered using the psd_model_mag data set. The number $m$ of test patterns and the tree height $h$ are fixed to $m=10,000,000$ and $h=9$, respectively. The ratio between test and test (chunks) is shown as black, thick line (a value close to 1 indicates a small overhead caused by the chunked processing).

\subsection{Modified Workflow}

While the modified workflow permits the use of buffer $k$-d trees for massive data sets that do not fit in the memories of the many-core devices, it might also induce a certain overhead compared to its original version due to the induced copy operations and reduced workload per kernel call. In addition, the "naive" use of multiple GPUs might exhibit a worse performance compared to filling the input queue with new queries on-the-fly. We now investigate both potential drawbacks.

Processing Leaves in Chunks: The main modification is the different processing of the leaf structure in case it does not fit in the device's memory. To evaluate the potential overhead caused by the additional copy operations (between host and device) during the execution of ProcessAllbuffers, we consider data set instances that still fit in memory and compare the runtimes of (1) the original workflow with (2) the workflow that is based on multiple chunks.

In Figure 3 , the outcome of this comparison is shown for a varying number $N$ of chunks and a varying number $n$ of training points. In all three cases, the number of test patterns is fixed to $m=10,000,000$. Further, the tree height is set to $h=9$ and a single GPU is used (i.e., bufferkdtree(1)). Two observations can be made: First, the training time is very small compared to the test time for all cases (even though the tree is constructed sequentially on the host). Second, the performance loss induced by the chunked processing is very small for almost any number $N$ of chunks (i.e., the ratio shown as black, thick line is close to $1)$. In particular, this is the case for smaller values of $N$; using more chunks naturally yields more overhead, which, however, decreases again if one increases the number of training and test patterns.

Thus, the runtimes of the new, chunked workflow are close to the one of the original approach - indicating that the overlapping compute-and-copy process successfully hides the additional overhead for the copy operations. 


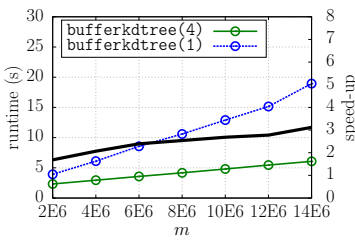

(a) psf_mag

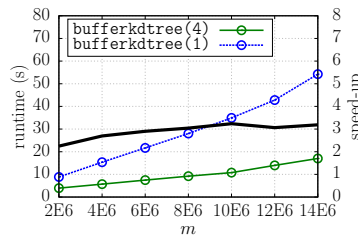

(b) psf_model_mag

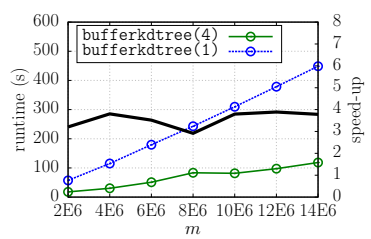

(c) all_mag

Figure 4: Runtime comparison for the test phase between bufferkdtree(1) and bufferkdtree (4), where the test queries are distributed uniformly among the devices for the latter one. The speed-up is shown as black, thick line (maximum 4).

Multi-Many-Core Processing: As outlined above, one can simply distribute the test queries to multiple devices to take advantage of the additional computational resources. Again, this can lead to a certain overhead, since invoking the procedure ProcessallBuffers becomes less efficient at the end of the overall processing (and this happens earlier in case the test queries are split into chunks). Similarly to the experiment provided above, we compare the efficiency of bufferkdtree(4) with the one of bufferkdtree(1), a standard single-device processing with all test patterns fitting on the GPU. For this sake, we consider $N=1$ leaf chunks (i.e., no modified processing of the leaves), $n=2 \cdot 10^{6}$ training patterns, and vary the number $m$ of test patterns.

The outcome of this experiment for three different data sets is shown in Figure 4 It can be seen that a suboptimal speed-up of about 2 is achieved in case a relatively small amount of test patterns is processed. However, as soon as the number of test patterns increases, the speed-up gets closer to 4, which depicts the maximum that can be achieved. Hence, the naive way of using all devices in a given workstation does not yield significant drawbacks as soon as large-scale scenarios are considered, which is the scope of this work.

\subsection{Large-Scale Applications}

To demonstrate the potential of the modified framework, we consider two largescale tasks: (1) the application of nearest neighbor models that are based on very large training sets and (2) large-scale density-based outlier detection.

Huge Nearest Neighbor Models: The first scenario addresses nearest neighbor models [12] that are based on very large training sets. Such models have been successfully been applied for various tasks in astronomy including the detection of distant galaxies or the estimation of physical parameters [192415].

For the experimental comparison we consider scenarios with both a large amount of training and test patterns. More precisely, we consider up to $n=$ $12 \cdot 10^{6}$ training points and up to $m=5 \cdot n$ test points given the psd_model mag data set. For both tree-based methods, appropriate tree depths are set beforehand (i.e., optimal ones w.r.t. the runtime needed in the test phase). The outcome of this comparison is shown in Figure 5. It can be seen that valuable 


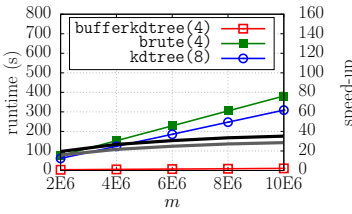

(a) $n=2 \cdot 10^{6}$

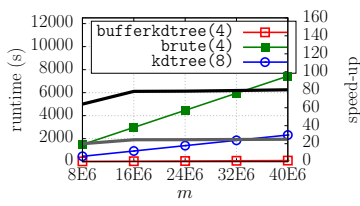

(d) $n=8 \cdot 10^{6}$

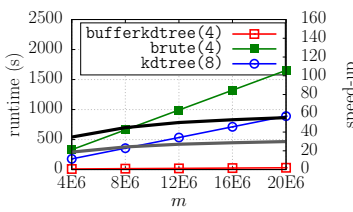

(b) $n=4 \cdot 10^{6}$

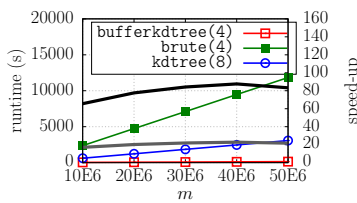

(e) $n=10 \cdot 10^{6}$

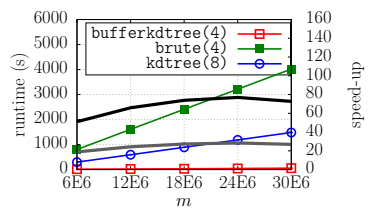

(c) $n=6 \cdot 10^{6}$

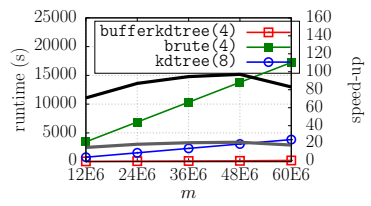

(f) $n=12 \cdot 10^{6}$

Figure 5: Runtime comparison for a varying number $n$ of training patterns given the psf_model_mag data set. The speed-ups of bufferkdtree over brute and kdtree are shown as thick black and gray lines, respectively. In each case, $m=n, \ldots, 5 n$ test patterns are considered for the bufferkdtree implementation; for both kdtree and brute, only $m=10^{6}$ were processed to obtain runtime estimates (which are plotted).

speed-ups can be achieved over both competitors. Further, the speed-ups generally become more significant the more patterns are processed. Note that for Figures (e) and (f), the bufferkdtree implementation automatically considers $N=3$ chunks (due to the training patterns exceeding the space reserved for them on the device), which results in a slightly worse performance for $m>30 \cdot 10^{6}$.

Large-Scale Proximity-Based Outlier Detection: As final use case, we consider large-scale proximity-based outlier detection. Various outlier scores have been proposed that are based on the computation of nearest neighbors. A typical one is to rank the points according to their average distance accordiing to their $k$ nearest neighbors, see, e.g., Tan et al. 25. Such techniques depict very promising tools in case many reference points are given in a moderate-sized feature space, which is precisely the case for many tasks in astronomy. Typically, these scores require the computation of the nearest neighbors for each of the reference points (known as all nearest neighbors problem). Naturally, this can quickly become very time-consuming.

To show the potential of our many-core implementation, we consider the crts data set described above (with $d=10$ features) and vary the number $n$ of reference points (here, we have $n=m$ for the full data set). We again compare the performances of all three competitors, where we consider both the runtime for the construction and the one for the query

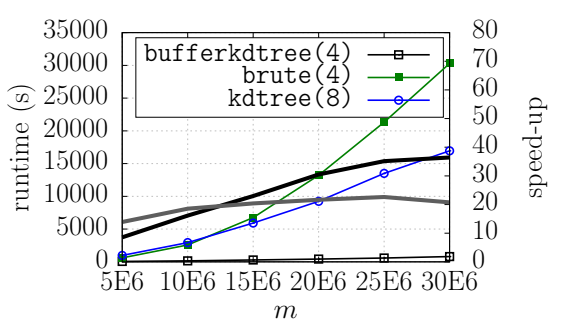

Figure 6: Large-Scale Outlier Detection 
phase. The outcome is shown in Figure 6. Note that the runtimes for both kdtree (cpu,8) and brute (gpu,4) depict estimates based on a reduced query due to the computational complexity (i.e., up to $n=30 \cdot 10^{6}$ reference points and a fixed query set of size $\hat{m}=1,000,000$ are considered; the runtime estimates w.r.t. to the full data set instances are plotted). For the bufferkdtree (gpu, 4) implementation, we fix $N=3$. It can be seen that the buffer $k$-d tree implementation yields valuable speed-ups and can successfully process the whole data set in a reasonable amount of time.

\section{Conclusions}

We provide a modified workflow for processing huge amounts of nearest neighbor queries using buffer $k$-d trees. The key idea is to process both the reference and the query points in chunks. While the latter is relatively easy to implement (even given several many-core devices), processing the reference points in chunks is more difficult. As shown in our work, one can effectively hide the overhead induced by the chunked processing of the reference points by interleaving the compute and copy operations. The experiments conducted on commodity hardware demonstrate that a single workstation is enough to efficiently process millions of reference and query points. Future work might address scenarios that are based on even larger reference sets (e.g., hundreds of billions of points), which could also necessitate the efficient construction of the buffer $k$-d tree. In addition, similar (chunked) buffering techniques might be useful to achieve efficient massively-parallel implementations for other techniques as well.

Acknowledgements. The authors would like to thank the Radboud Excellence Initiative of the Radboud University Nijmegen and Nvidia for its support and generous hardware donations (FG), the Danish Industry Foundation through the Industrial Data Analysis Service (CI, CO), the The Danish Council for Independent Research $\mid$ Natural Sciences through the project Surveying the sky using machine learning (CI), and ACP, IUCAA, IUSSTF, and NSF (AM).

\section{References}

1. Bentley, J.: Multidimensional binary search trees used for associative searching. Communications of the ACM 18(9), 509-517 (1975)

2. Beygelzimer, A., Kakade, S., Langford, J.: Cover trees for nearest neighbor. In: Proceedings of the 23rd International Conference on Machine Learning. pp. 97104. ACM (2006)

3. Blum, M., Floyd, R.W., Pratt, V., Rivest, R.L., Tarjan, R.E.: Time bounds for selection. Journal of Computer and System Sciences 7(4), 448-461 (1973)

4. Bustos, B., Deussen, O., Hiller, S., Keim, D.: A graphics hardware accelerated algorithm for nearest neighbor search. In: Computational Science - ICCS 2006. Lecture Notes in Computer Science, vol. 3994, pp. 196-199. Springer (2006) 
5. Cayton, L.: Accelerating nearest neighbor search on manycore systems. In: Proceedings of the 2012 IEEE 26th International Parallel and Distributed Processing Symposium. pp. 402-413. IEEE (2012)

6. Djorgovski, S.G., Drake, A.J., Mahabal, A.A., Graham, M.J., Donalek, C., Williams, R., Beshore, E.C., Larson, S.M., Prieto, J., Catelan, M., Christensen, E., McNaught, R.H.: The catalina real-time transient survey. The First Year of MAXI: Monitoring Variable X-ray Sources (2011)

7. Drake, A.J., Djorgovski, S.G., Mahabal, A., Beshore, E., Larson, S., Graham, M.J., Williams, R., Christensen, E., Catelan, M., Boattini, A., Gibbs, A., Hill, R., Kowalski, R.: First results from the catalina real-time transient survey. The Astrophysical Journal 696(1), 870-884 (2009)

8. Faraway, J., Mahabal, A., Sun, J., Wang, X., Yi, W., Zhang, L.: Modeling light curves for improved classification. eprint arXiv:1401.3211 (2014)

9. Friedman, J., Bentley, J., Finkel, R.: An algorithm for finding best matches in logarithmic expected time. ACM Transactions on Mathematical Software 3(3), 209-226 (1977)

10. Garcia, V., Debreuve, E., Nielsen, F., Barlaud, M.: K-nearest neighbor search: Fast GPU-based implementations and application to high-dimensional feature matching. In: Proceedings of the 17th IEEE International Conference on Image Processing. pp. 3757-3760. IEEE (2010)

11. Gieseke, F., Heinermann, J., Oancea, C., Igel, C.: Buffer k-d trees: Processing massive nearest neighbor queries on GPUs. In: Proceedings of the 31st International Conference on Machine Learning (ICML 2014). JMLR W\&CP. vol. 32, pp. 172-180 (2014)

12. Hastie, T., Tibshirani, R., Friedman, J.: The Elements of Statistical Learning. Springer, 2 edn. (2009)

13. Heinermann, J., Kramer, O., Polsterer, K.L., Gieseke, F.: On GPU-based nearest neighbor queries for large-scale photometric catalogs in astronomy. In: KI 2013: Advances in Artificial Intelligence, Lecture Notes in Computer Science, vol. 8077, pp. 86-97. Springer (2013)

14. Indyk, P., Motwani, R.: Approximate nearest neighbors: Towards removing the curse of dimensionality. In: Proceedings of the 30th Annual ACM Symposium on Theory of Computing. pp. 604-613. ACM (1998)

15. Kremer, J., Gieseke, F., Steenstrup Pedersen, K., Igel, C.: Nearest neighbor density ratio estimation for large-scale applications in astronomy. Astronomy and Computing 12, 67-72 (2015)

16. Mahabal, A.A., Djorgovski, S.G., Drake, A.J., Donalek, C., Graham, M.J., Williams, R.D., Chen, Y., Moghaddam, B., Turmon, M., Beshore, E., Larson, S.: Discovery, classification, and scientific exploration of transient events from the catalina real-time transient survey. Bulletin of the Astronmical Society of India 39(3), 387-408 (2011)

17. Nakasato, N.: Implementation of a parallel tree method on a GPU. Journal of Computational Science 3(3), 132-141 (2012)

18. Pan, J., Manocha, D.: Fast GPU-based locality sensitive hashing for k-nearest neighbor computation. In: Proceedings of the 19th ACM SIGSPATIAL International Conference on Advances in Geographic Information Systems. pp. 211-220. ACM (2011)

19. Polsterer, K., Zinn, P., Gieseke, F.: Finding new high-redshift quasars by asking the neighbours. Monthly Notices of the Royal Astronomical Society 428(1), 226-235 (2013) 
20. Qiu, D., May, S., Nüchter, A.: GPU-accelerated nearest neighbor search for 3D registration. In: Proceedings of the 7th International Conference on Computer Vision Systems. pp. 194-203. Springer (2009)

21. Richards, J.W., Starr, D.L., Butler, N.R., Bloom, J.S., Brewer, J.M., Crellin-Quick, A., Higgins, J., Kennedy, R., Rischard, M.: On machine-learned classification of variable stars with sparse and noisy time-series data. The Astrophysical Journal 733(1) (2011)

22. Scarpino, M.: OpenCL in Action: How to Accelerate Graphics and Computation. Manning (2012)

23. Sismanis, N., Pitsianis, N., Sun, X.: Parallel search of k-nearest neighbors with synchronous operations. In: IEEE Conference on High Performance Extreme Computing. pp. 1-6. IEEE (2012)

24. Stensbo-Smidt, K., Igel, C., Zirm, A., Steenstrup Pedersen, K.: Nearest neighbour regression outperforms model-based prediction of specific star formation rate. In: IEEE International Conference on Big Data 2013. pp. 141-144. IEEE (2013)

25. Tan, P.N., Steinbach, M., Kumar, V.: Introduction to Data Mining. AddisonWesley (2005)

26. Wang, W., Cao, L.: Parallel k-nearest neighbor search on graphics hardware. In: Proceedings of the 2010 3rd International Symposium on Parallel Architectures, Algorithms and Programming. pp. 291-294. IEEE (2010) 